\title{
Pronóstico de engelamiento y ondas de montaña mediante modelos mesoescalares orientado a mejorar la seguridad aérea
}

https://doi.org/10.31978/639-19-010-0.273

\author{
Sergio Fernández González ${ }^{1}$ (sfernandezg@aemet.es) \\ Andrés Merino Suances ${ }^{2}$ (amers@unileon.es) \\ Pedro Bolgiani³ (pbolgian@ucm.es)
}

\begin{abstract}
${ }^{1}$ AEMET / Dirección de Producción e Infraestructuras / Departamento de Producción
${ }^{2}$ Universidad de León / Grupo de Física de la Atmósfera, Instituto de Medio Ambiente

${ }^{3}$ Universidad Complutense de Madrid / Departamento de Física de la Tierra y Astrofísica
\end{abstract}

\begin{abstract}
RESUMEN
La turbulencia y la formación de hielo en las aeronaves son dos de los fenómenos meteorológicos más peligrosos en la seguridad de la aviación. Por lo tanto, se recomienda encarecidamente evitar las áreas con alta probabilidad de episodios de formación de hielo a lo largo de las rutas de llegada a, y salida de, los aeropuertos. Este problema es relativamente común en el aeropuerto internacional Madrid-Barajas Adolfo Suárez (LEMD), por lo que es necesario caracterizar estos episodios y mejorar su pronóstico.

En este trabajo se ha utilizado el modelo Weather Research and Forecasting (WRF) para la simulación de episodios de ondas de montaña previamente observados. También se han usado herramientas de teledetección tanto para la verificación de las salidas del modelo como para su aplicación al nowcasting o predicción en tiempo real. Esta información puede resultar de gran utilidad en la gestión de las operaciones aeroportuarias, con el objetivo de evitar áreas con riesgo de turbulencia y engelamiento.
\end{abstract}

PALABRAS CLAVE: ondas de montaña; engelamiento; cizalladura; seguridad aérea; WRF.

\section{INTRODUCCIÓN}

Las ondas de montaña se desarrollan a sotavento de las barreras orográficas cuando el flujo de viento es perpendicular a las mismas, caracterizándose por sucesivas corrientes ascendentes y descendentes (ALLEN y Vincent, 1995). Al incidir sobre la montaña con la suficiente energía cinética, la masa de aire es obligada a ascender, mientras que a sotavento se produce una corriente descendente. Para que aparezcan ondas de montaña se requiere una atmósfera estable o neutra (PETERSEN et al., 2005). Estos movimientos ondulatorios se pueden propagar a mayor o menor distancia a sotavento del sistema montañoso, en función de la intensidad del viento y la estabilidad atmosférica (BRoutman et al., 2001). De existir suficiente humedad, en la corriente ascendente se puede generar nubosidad asociada a las ondas de montaña, como son las nubes lenticulares o de tipo rotor. Las ondas de montaña se pueden propagar hasta grandes distancias a sotavento del sistema montañoso, y pueden alcanzar alturas considerables. Sin embargo, su anchura es reducida al coincidir con la región en la que aparece la corriente ascendente. Esto provoca que el tiempo de residencia de los hidrometeoros en las nubes lenticulares sea reducido, dificultando la formación de cristales de hielo. Por tanto, es frecuente la aparición de agua líquida sobreenfriada en las ondas de montaña, especialmente durante 
el periodo invernal, con el correspondiente riesgo de engelamiento para las aeronaves que atraviesen estas nubes.

El mayor interés en el estudio de las ondas de montaña está relacionado con la seguridad aérea. Entre los factores meteorológicos que más afectan a la seguridad aérea destacan el engelamiento, la cizalladura, la turbulencia, y la visibilidad reducida en niveles bajos (Moreno y GiL, 2003). En el caso de las ondas de montaña, el riesgo de turbulencia generado por las corrientes verticales se combina con el riesgo de engelamiento, sobre todo durante el invierno, debido a la presencia de agua líquida sobreenfriada en la nubosidad asociada. La investigación de estos episodios en las proximidades de aeropuertos se hace imprescindible debido a los numerosos accidentes registrados en las últimas décadas asociados a engelamiento (BERNSTEIN et al., 2005). En el caso de no haber humedad suficiente para que se forme nubosidad, existe el peligro de que se produzca turbulencia en aire claro, que es un riesgo importante para la seguridad aérea al no existir indicadores de su presencia.

Aunque la formación de ondas de montaña y engelamiento asociado a las mismas es relativamente común, el pronóstico y la observación de estos episodios están lejos de ser perfectos. Para simular de forma precisa estos fenómenos meteorológicos es necesario utilizar modelos mesoescalares con una elevada resolución tanto espacial como temporal. Estudios anteriores han demostrado la eficacia del modelo mesoescalar WRF en la detección de ondas de montaña y engelamiento asociado a las mismas en el centro de la península ibérica (FERNÁNDEZ GONZÁlez et al., 2014; BolgiANI et al., 2018), siendo imprescindible una elevada resolución espacial para simular adecuadamente estos episodios.

La observación de los episodios de ondas de montaña es una tarea compleja. Para alcanzar esta meta, una opción válida es el uso de teledetección. Las imágenes captadas por satélites meteorológicos permiten la detección de los movimientos ondulatorios a través de la nubosidad asociada, así como las variaciones en la concentración de vapor de agua. En concreto, el satélite METEOSAT de segunda generación (MSG) es una herramienta muy adecuada para el estudio de diversos tipos de nubosidad, entre los que se incluyen las nubes lenticulares y de tipo rotor, características de las ondas de montaña. Al disponer de distintos canales espectrales, permite detectar diferentes propiedades de absorción que presenta en esta longitud de onda el hielo y el agua líquida, lo que hace posible inferir la fase en la que se encuentran los hidrometeoros localizados en el techo nuboso (SETVÁK et al., 2003).

El principal objetivo de este trabajo es caracterizar las variables meteorológicas asociadas a la formación de ondas de montaña al sur de la sierra de Guadarrama durante episodios de viento con componente noroeste. Así mismo, se pretenden identificar herramientas útiles para mejorar el pronóstico de estos episodios, como los modelos mesoescalares y las técnicas de teledetección.

\section{METODOLOGÍA}

En esta sección se va a realizar la descripción de las principales herramientas utilizadas en este estudio. Finalmente, se detallará la base de datos utilizada.

\subsection{Satélite MSG}

En este trabajo se han utilizado imágenes del satélite MSG con una resolución temporal de 15 minutos. El estudio se ha centrado en la detección de ondas de montaña mediante el canal visible de alta resolución (HRV) durante el periodo diurno (capaz de captar radiación electromagnética en el intervalo 0,4-1,1 $\mu \mathrm{m}$ ), mientras que para el periodo nocturno se ha usado el canal de $7,3 \mu \mathrm{m}$, el cual se encuentra centrado en la banda de absorción del vapor de agua, siendo capaz de inferir la humedad en la media y baja troposfera.

Además, se ha utilizado la composición de colores RGB (Red-Green-Blue) conocida como day natural colors (LENSKY y ROSENFELD, 2008), que se compone de los canales de 1,6 $\mu \mathrm{m}, 0,8 \mu \mathrm{m}$ y 0,6 $\mu \mathrm{m}$ en los 
colores rojo, verde y azul respectivamente, y permite discernir la fase en la que se encuentran los hidrometeoros en el techo nuboso. En las imágenes generadas mediante esta composición de colores las nubes en la fase líquida aparecerán en color blanco, mientras que aquellas en fase de hielo estarán coloreadas de azul. Esto se debe a la fuerte absorción de hielo en la banda de 1,6 $\mu \mathrm{m}$ (rojo en el RGB), que da un color azulado o cian, mientras que las nubes en fase líquida tienen un fuerte reflejo en las tres bandas, dando lugar a un color blanquecino.

\subsection{Modelo WRF}

El modelo que se ha utilizado en esta investigación es el Weather Research and Forecasting (WRF) versión 3.7.1 (SKAMAROCK y KLEMP, 2008). Se ha elegido por ser un modelo mesoescalar no hidrostático que permite realizar simulaciones con una elevada resolución tanto espacial como temporal. Asimismo, dispone de varias parametrizaciones seleccionables. Esto permite que, según la zona de estudio sobre la que se vaya a aplicar la modelización y el fenómeno meteorológico a estudiar, se pueda analizar qué parametrización se ajusta mejor a las particularidades del emplazamiento.

Se han realizado simulaciones de 24 horas (considerando las 6 primeras horas como spin-up del modelo, por lo que no se usan para el análisis) para cada episodio analizado. Las condiciones iniciales que se han usado para lanzar el modelo fueron extraídas del modelo global GFS, con una resolución espacial de $0,25^{\circ}$, y una resolución temporal de 3 horas. Se han definido 3 dominios anidados con resoluciones horizontales de 9,3 y $1 \mathrm{~km}$, respectivamente (figura 1.a). Cada dominio tiene $121 \times 121$ puntos de malla, con 40 niveles verticales sigma con una resolución progresiva (más elevada en los niveles bajos de la troposfera).
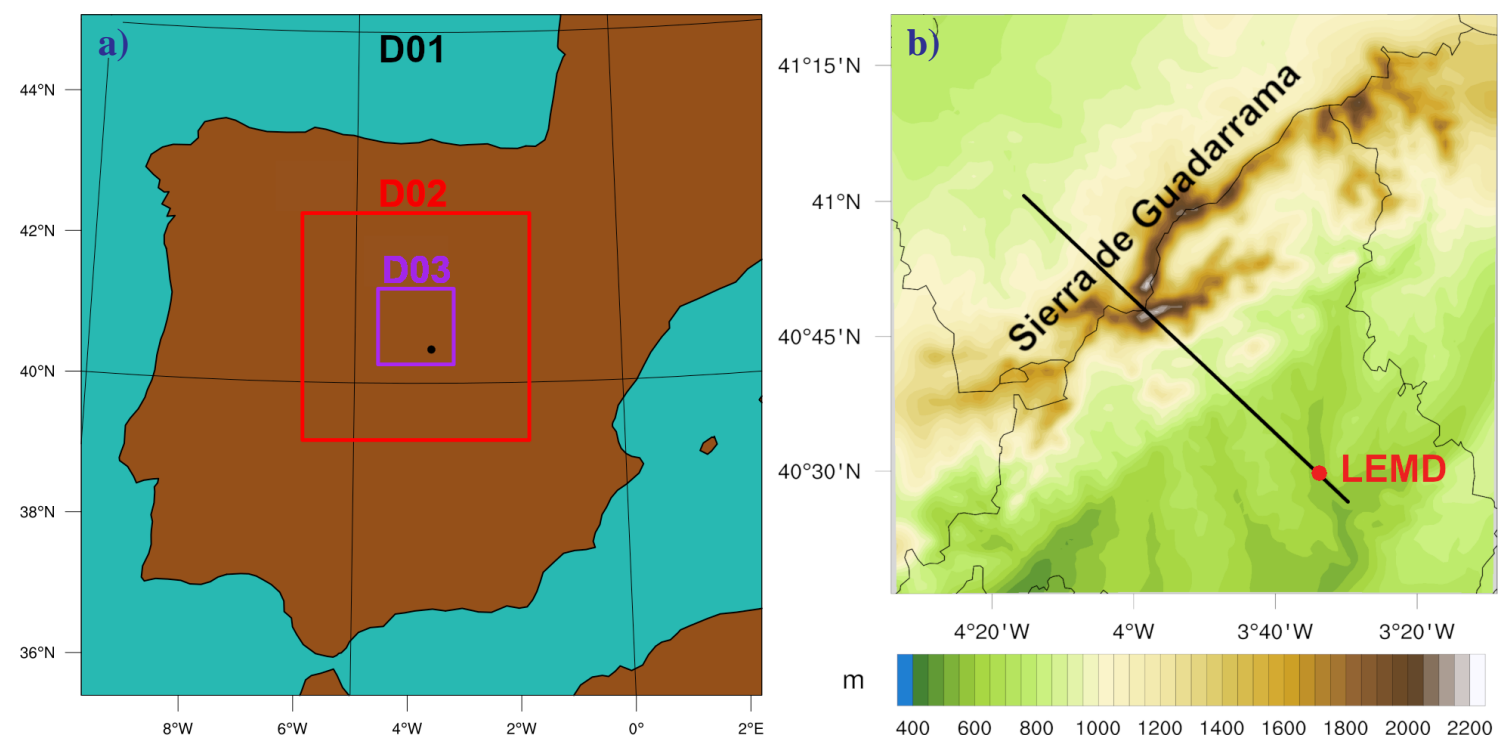

Figura 1. a) Configuración de los dominios del modelo WRF. b) Orografía del dominio 3.

Respecto a la configuración de las parametrizaciones de la radiación del modelo WRF, se ha usado el esquema definido por DudHIa (1989) y el Rapid Radiative Transfer Model descrito por Mlawer et al. (1997) para la radiación de onda corta y onda larga, respectivamente. La parametrización microfísica utilizada fue la desarrollada por THOMPSON et al. (2008), mientras que la parametrización de SMIRNova et al. (2000) fue seleccionada como modelo de superficie terrestre, y la de NAKANISHI y NIINO (2006) para la parametrización de la capa límite planetaria. Finalmente, la parametrización de cúmulos definida por KAIN (2004) se estableció para el dominio 1, mientras que en los dominios 2 y 3 los cúmulos se resuelven de forma explícita. Esta combinación de parametrizaciones fue previamente utilizada por BolgIANI et al. (2018) en la simulación de ondas de montaña en el centro de la península ibérica. 


\subsection{Base de datos}

A lo largo del invierno 2017/2018 se han analizado las imágenes de los canales del MSG anteriormente descritos para detectar la formación de ondas de montaña a sotavento del sistema Central, que pudieran afectar a las maniobras de aterrizaje o despegue desde el aeropuerto internacional Madrid-Barajas Adolfo Suárez (LEMD). Finalmente, se han seleccionado 10 días (tabla 1) que posteriormente se simularon con el modelo WRF para analizar en detalle las variables meteorológicas relacionadas con la formación de ondas de montaña. Se han seleccionado los 8 episodios en los que la formación de ondas de montaña fue más evidente. Además, se añadieron a la base de datos 2 eventos en los que, a pesar de que el viento de componente noroeste era favorable a la formación de ondas de montaña, no se llegaron a detectar a través de las imágenes satelitales.

\begin{tabular}{|rl|c|}
\hline \multicolumn{2}{|c|}{ Días con ondas de montaña observadas } & Días sin ondas de montaña observadas \\
\hline $2017 / 12 / 02$ & $2018 / 01 / 02$ & $2017 / 12 / 12$ \\
\hline $2017 / 12 / 27$ & $2018 / 01 / 26$ & $2018 / 02 / 22$ \\
\hline $2017 / 12 / 29$ & $2018 / 02 / 03$ & \\
\hline $2018 / 01 / 01$ & $2018 / 03 / 07$ & \\
\hline
\end{tabular}

Tabla 1. Listado de los días con/sin ondas de montaña que conforman la base de datos.

\section{ZONA DE ESTUDIO}

Las investigaciones desarrolladas en este trabajo se centran en la sierra de Guadarrama, situada en la zona centro de la península ibérica (figura 1.b). Su punto más alto es el Peñalara, alcanzando una altura de 2428 metros sobre el nivel del mar (msnm). El valle del Lozoya divide la sierra de Guadarrama en dos, abriéndose entre montañas con alturas superiores a $2000 \mathrm{~m}$ al norte, y $1700 \mathrm{~m}$ al sur.

Este sistema montañoso se sitúa a escasas decenas de kilómetros de LEMD, por lo que en condiciones de meteorología adversa puede llegar a influir en las operaciones de aterrizaje y despegue, especialmente cuando el viento a la altura de las cimas de la sierra de Guadarrama presenta componente noroeste (perpendicular a la sierra de Guadarrama), pudiéndose formar ondas de montaña a sotavento. Cuando el aeropuerto LEMD opera con configuración sur (viento en superficie de componente sur) puede existir riesgo de atravesar la región donde se forman las ondas de montaña durante la aproximación, mientras que cuando hay configuración norte, el riesgo se produce durante la fase de salida.

\section{RESULTADOS}

La sección de resultados se divide en la observación de los episodios mediante los distintos canales del satélite MSG, y en la simulación de los mismos eventos mediante el modelo WRF. Los resultados mostrados se van a centrar en los episodios de los días 2 y 12 de diciembre de 2017. En el episodio del día 2 se apreció de forma nítida la formación de nubosidad asociada a las ondas de montaña en las imágenes satelitales, mientras que durante el día 12, a pesar de existir viento de componente noroeste, no se apreció en el satélite la formación de ondas de montaña.

\subsection{Imágenes obtenidas a partir del satélite MSG}

El uso de las imágenes procedentes de los distintos canales del satélite MSG puede ser de gran utilidad tanto en la detección de episodios de ondas de montaña como en el nowcasting aplicado a la predicción operativa. El canal del satélite MSG en el que más nítidamente se detecta la nubosidad asociada a las ondas de montaña 
es el canal HRV. Como se puede observar en la figura 2.a, la aparición de bandas nubosas paralelas a una barrera orográfica y a sotavento de la misma es una señal inequívoca de que en esa región existe riesgo de turbulencia, y puede ser conveniente que las aeronaves no atraviesen dicha zona. En la figura 2.b se muestra una imagen del canal HRV de un día sin ondas de montaña, en el que a pesar de haber viento de componente norte (como indican las nubes bajas de carácter orográfico retenidas en la cara norte del sistema Central), no se llegan a formar nubes asociadas a ondas de montaña a sotavento.

Sin embargo, el canal HRV presenta los inconvenientes de que solo capta imágenes durante el periodo diurno, y que solo detecta ondas de montaña cuando se forma nubosidad asociada. Por ello, se propone el uso del canal de 7,3 $\mu \mathrm{m}$ que, centrado en la banda de absorción del vapor de agua en niveles medios y bajos de la troposfera, permite detectar regiones con mayor/menor contenido de humedad. De esta forma podemos realizar un seguimiento continuo durante todo el día. Además, el canal de 7,3 $\mu \mathrm{m}$ puede ser capaz de detectar ondas de montaña incluso cuando no existe nubosidad asociada, ya que aparecerán bandas alternas brillantes (alto contenido de vapor de agua, ligadas a las corrientes ascendentes) y oscuras (bajo contenido de vapor de agua, relacionadas con las corrientes descendentes). Por tanto, en este canal se podría detectar incluso la presencia de turbulencia en aire claro.

En las figuras 3.a y 3.b se han representado imágenes del canal de 7,3 $\mu$ m para un día con ondas de montaña, el que se aprecia la propagación de las mismas a sotavento del sistema Central desde primera hora de la mañana, extendiéndose durante todo el día. Sin embargo, en el caso del día 12 de diciembre de 2017 (figura 3.c y 3.d), aunque a primera hora se intuyen una o dos ondas de montaña a sotavento del sistema Central (sin propagarse más hacia el sur), conforme avanza el día desaparecen.

Adicionalmente, se propone el uso de la composición de colores denominada day natural colors (LENSKY y ROSENFELD, 2008), que permite diferenciar la nubosidad cuyo techo se encuentra en fase líquida (coloreadas de blanco) de la nubosidad cuyo techo está en fase sólida (en color cian, al igual que la nieve o el hielo en superficie). En la figura 4.a se puede ver como la nubosidad asociada a las ondas de montaña al sur del sistema Central se halla en fase líquida. Por tanto, de encontrarnos por encima de la isocero, podría haber riesgo de engelamiento además de la turbulencia causada por las ondas de montaña, por lo que sería conveniente evitar esta región en las maniobras de aproximación y salida de LEMD. Al igual que en el caso del canal HRV, a mediodía del 12 de diciembre de 2017 no se aprecia nubosidad al sur del sistema Central (figura 4.b). La composición de colores day natural colors ya ha sido testada en episodios de engelamiento en la aproximación a LEMD con resultados satisfactorios (BoLGIANI et al., 2018), confirmando su utilidad en el nowcasting de episodios de engelamiento asociado a ondas de montaña.
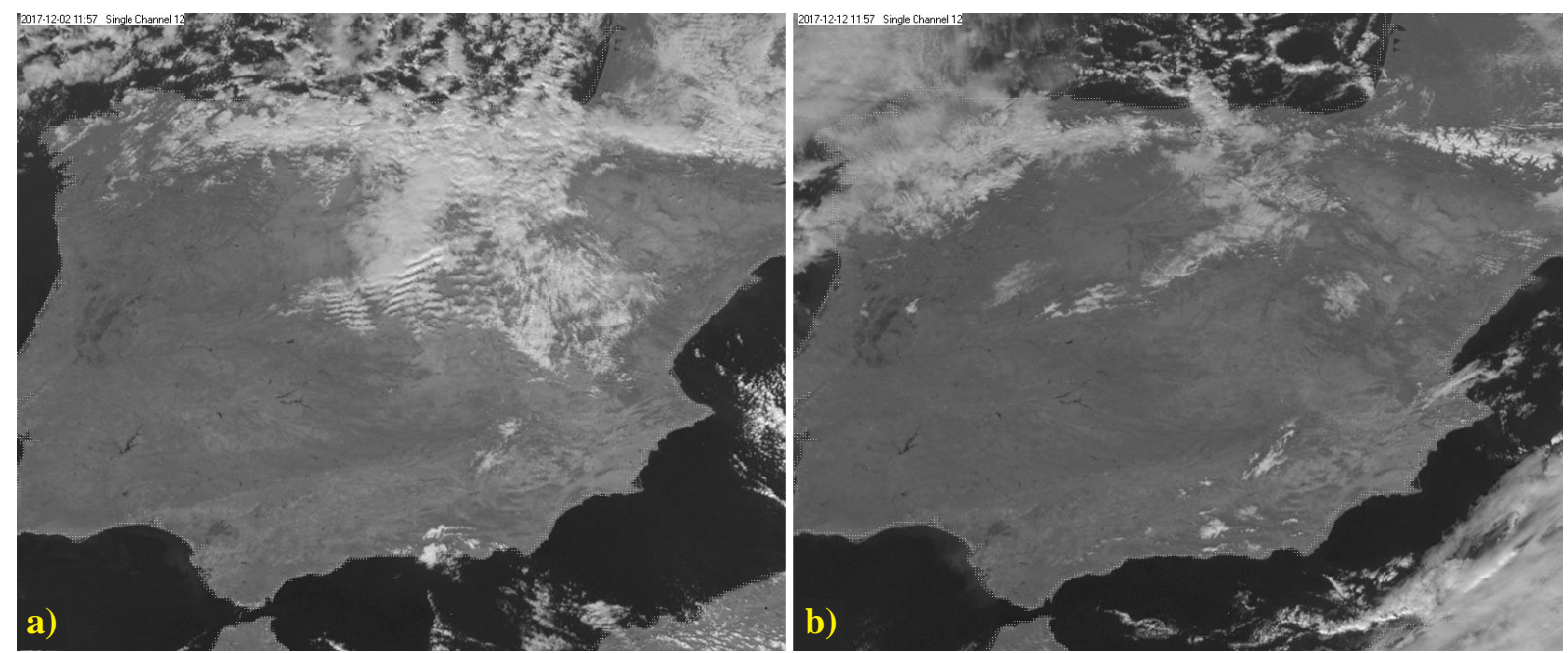

Figura 2. Imágenes obtenidas del canal visible de alta resolución del satélite MSG a las 11:57 UTC de los días a) 2 de diciembre de 2017 y b) 12 de diciembre de 2017. 

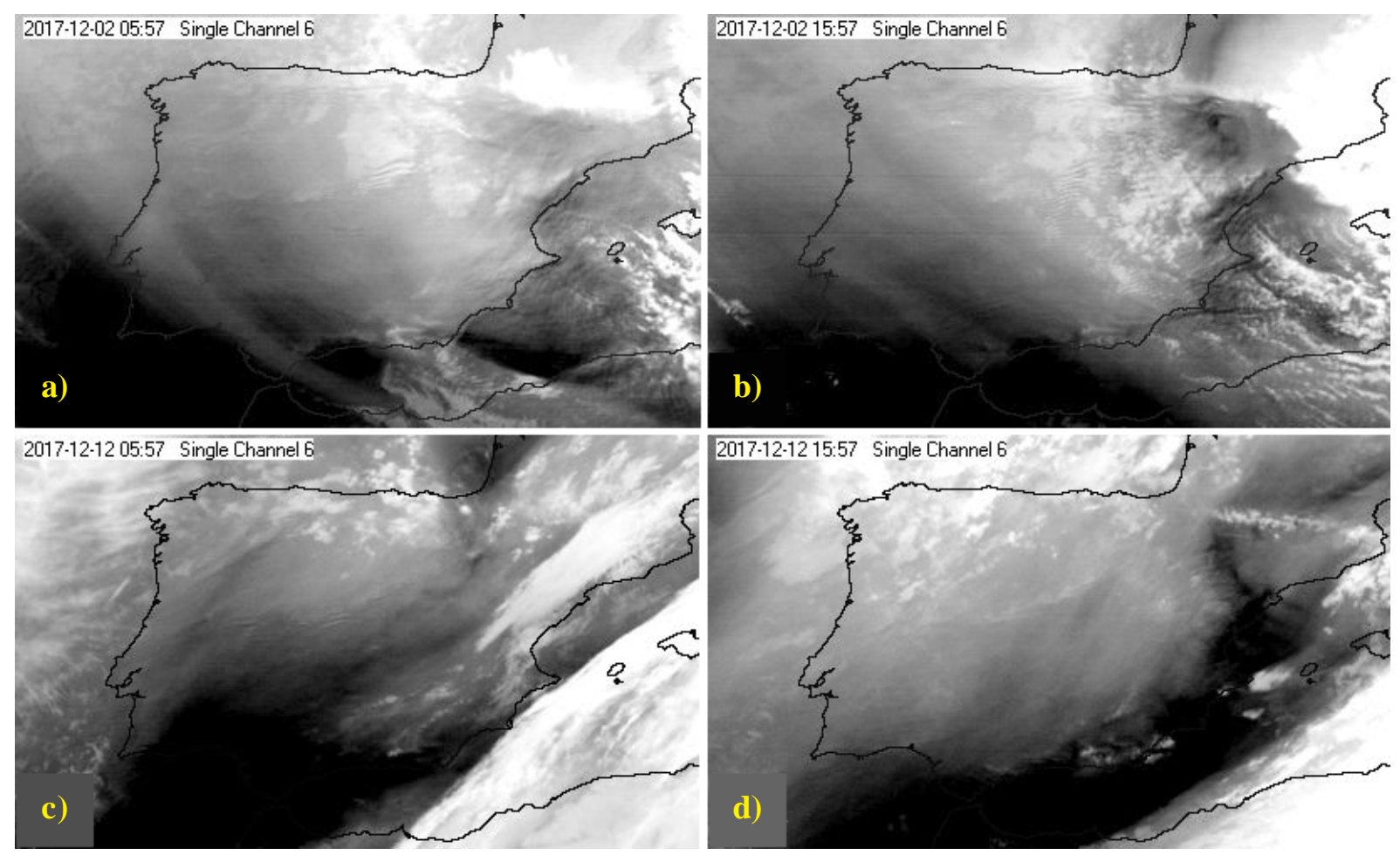

Figura 3. Imágenes procedentes del canal de 7,3 $\mu \mathrm{m}$ del satélite MSG a las a) 05:57 y b) 15:57 UTC del día 2 de diciembre de 2017, y a las c) 05:57 y d) 15:57 UTC del día 12 de diciembre de 2017.
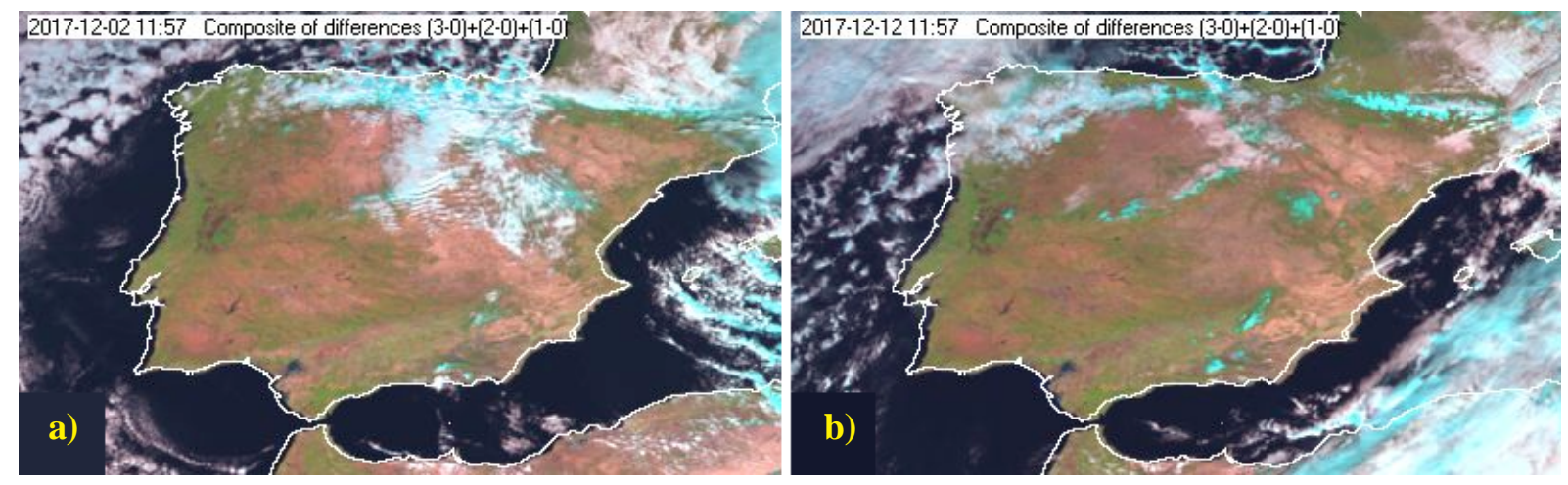

Figura 4. Imágenes de la composición de colores day natural colors del satélite MSG a las 11:57 UTC de los días a) 2 de diciembre de 2017 y b) 12 de diciembre de 2017.

\subsection{Simulaciones realizadas con el modelo WRF}

Una vez detectadas las ondas de montaña mediante las imágenes satelitales, se ha procedido a simular los episodios seleccionados en la base de datos mediante el modelo mesoescalar WRF. En esta sección se van a representar las simulaciones de los mismos dos días tratados en la sección anterior ( 2 de diciembre de 2017: ejemplo de día con ondas de montaña; 12 de diciembre de 2017: ejemplo de día sin ondas de montaña).

Tras realizar distintas pruebas, se ha llegado a la conclusión de que se requiere una resolución espacial de $1 \mathrm{~km}$ para simular correctamente las ondas de montaña, ya que, en el dominio de $3 \mathrm{~km}$, aunque las corrientes verticales asociadas a las ondas de montaña sí son detectadas, no se simula correctamente la nubosidad asociada a las ondas. Por tanto, a continuación, se va a proceder a analizar las salidas del dominio 3 del modelo WRF (resolución espacial de $1 \mathrm{~km}$ ) para los campos meteorológicos más relacionados con la formación de ondas de montaña. 
En primer lugar, se ha analizado el viento a $2500 \mathrm{msnm}$ simulado por el modelo WRF en el dominio 3. Se ha fijado esta altura ya que coincide aproximadamente con la altitud de las cimas de la sierra de Guadarrama. Las figuras 5.a y 5.b corresponden al 2 de diciembre de 2017, día en el que se observaron ondas de montaña en las imágenes de satélite. Se puede apreciar como el viento a $2500 \mathrm{msnm}$ fue superior a $30 \mathrm{kt}$ y tuvo componente norte durante toda la jornada, formándose a sotavento bandas alternas con mayor y menor velocidad, que ya sugieren la formación de ondas de montaña.

Respecto al día 12 de diciembre de 2017 (figuras 5.c y 5.d), el viento a 2500 msnm es de componente noroeste, disminuyendo en intensidad a lo largo del día desde unos $20 \mathrm{kt}$ a primeras horas (cuando en el satélite se intuyen ondas de montaña, aunque no muy definidas), a 5-10 kt por la tarde (cuando ya no se registraban ondas de montaña en las imágenes de satélite). En el episodio de engelamiento asociado a ondas de montaña analizado por Bolgiani et al. (2018) se registró una velocidad de viento de unos $40 \mathrm{kt}$ a $3300 \mathrm{msnm}$, lo que es coherente con los resultados de este trabajo. Los resultados obtenidos a partir de las simulaciones con el modelo WRF de los episodios de la base de datos indican que en la sierra de Guadarrama se requieren vientos a $2500 \mathrm{msnm}$ con velocidades superiores a $20 \mathrm{kt}$ y con componente $\mathrm{NW}$ (aunque se pueden formar desde componente $\mathrm{W}$ a componente $\mathrm{N}$ ) para que se generen ondas de montaña a sotavento.
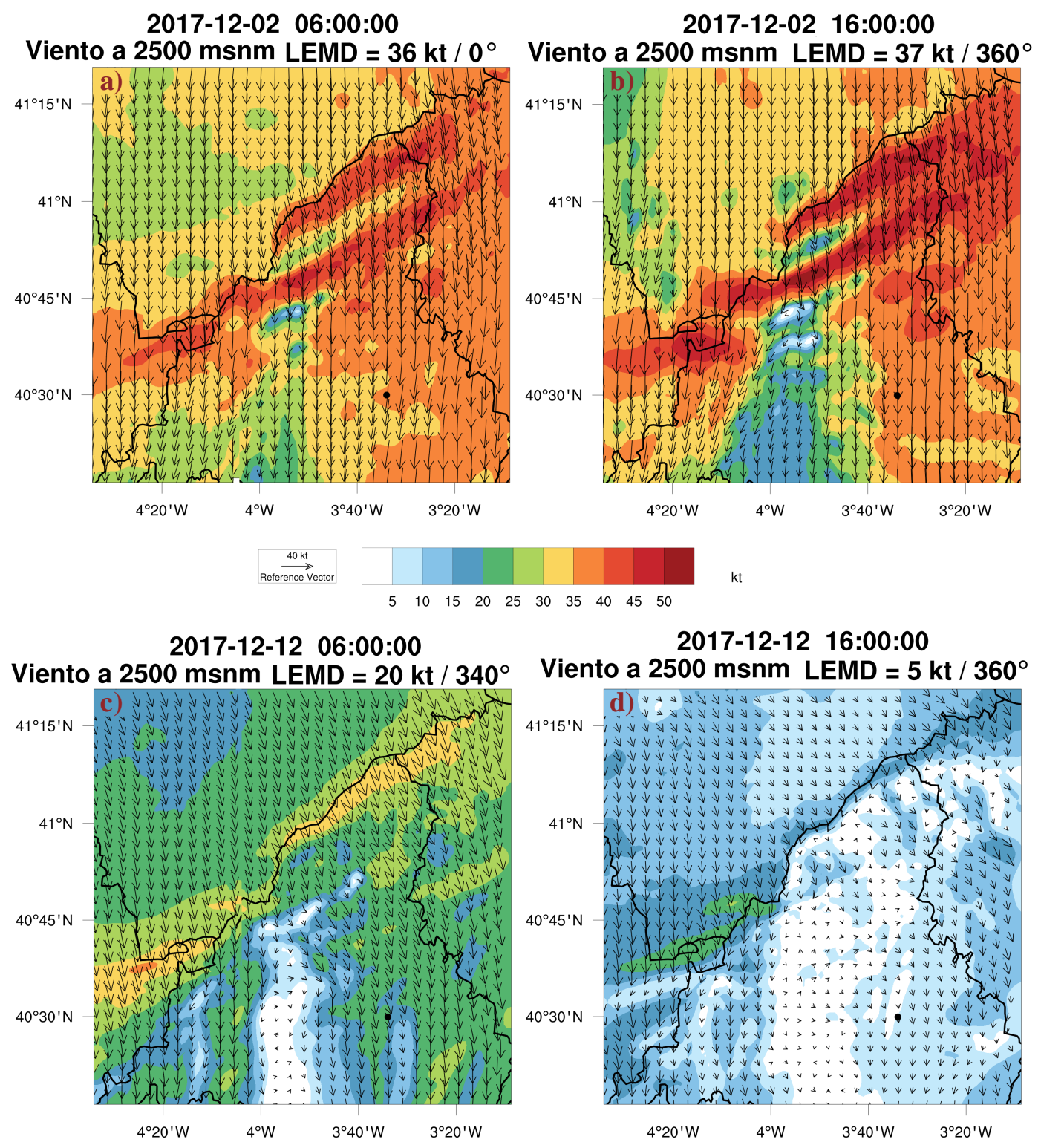

Figura 5. Viento a $2500 \mathrm{msnm}$ simulado por el modelo WRF en el dominio 3 a las 06:00 y 16:00 UTC del día 2 de diciembre de 2017 (a y b), y del día 12 de diciembre de 2017 (c y d).

El punto negro representa la localización del aeropuerto LEMD. 

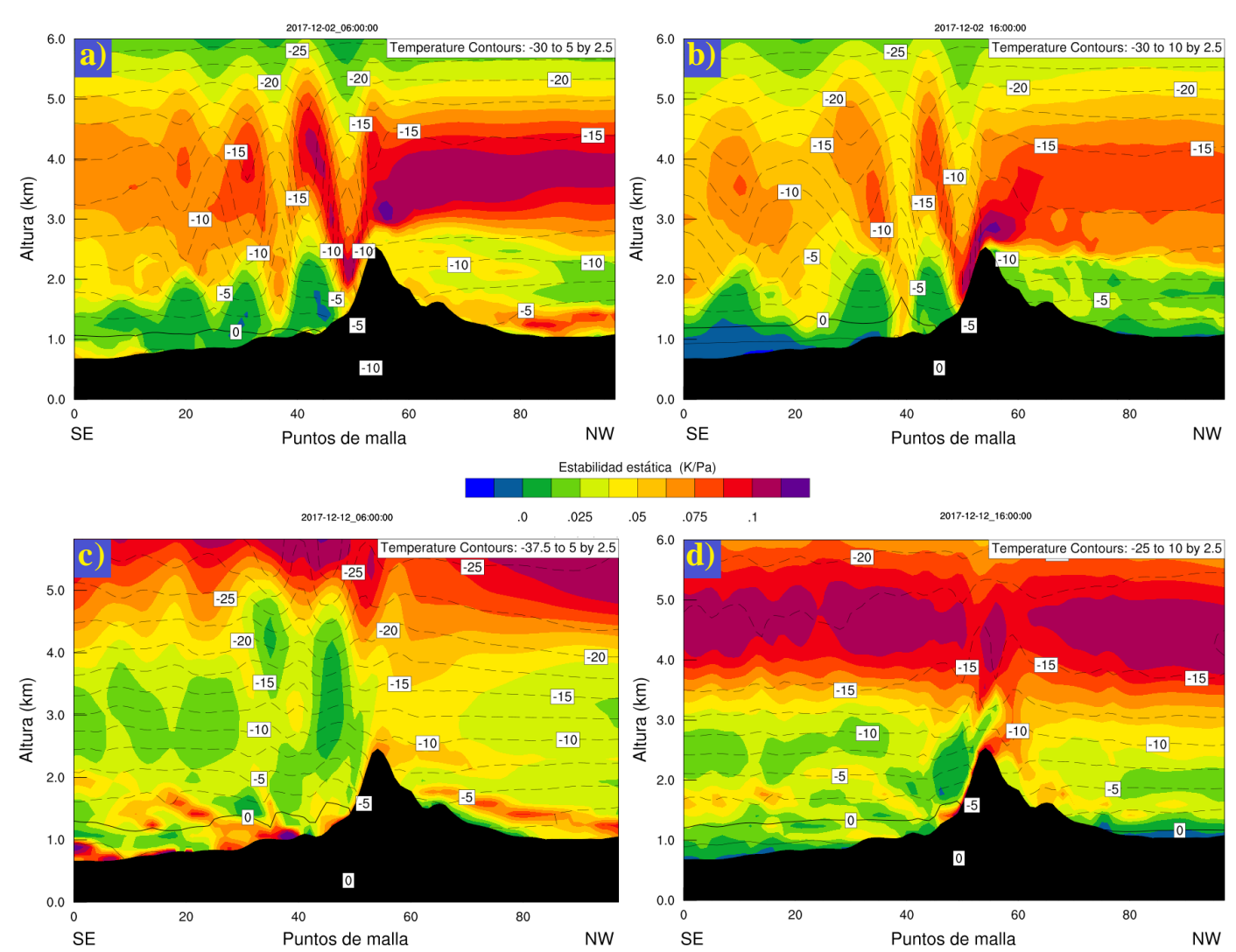

Figura 6. Corte vertical de la estabilidad estática estimada por el modelo WRF en el dominio 3 a las 06:00 y 16:00 UTC del día 2 de diciembre de 2017 (a y b, respectivamente), y a las 06:00 y 16:00 UTC del día 12 de diciembre de 2017 (c y d).

Después se ha analizado la estabilidad estática mediante un corte vertical (línea negra de la figura 1.b) de los $6 \mathrm{~km}$ más bajos de la troposfera en el dominio 3 del WRF. Como se puede ver en las figuras 6.a y 6.b correspondientes al 2 de diciembre de 2017, en niveles medios (entre 2500 y $6000 \mathrm{msnm}$ ) existe ligera estabilidad estática (valores en torno a $0,075 \mathrm{~K} / \mathrm{Pa}$ ), lo que es propicio a la formación de ondas de montaña (FERNÁNDEZ GoNZÁLEZ et al., 2014). Las oscilaciones de las isotermas a sotavento de la sierra de Guadarrama sugieren la formación de ondas de montaña. Sin embargo, en el día 12 de diciembre de 2017 predominan condiciones de estabilidad estática neutra, menos favorable para la propagación de ondas de montaña.

El modelo WRF permite estimar la componente vertical del viento, variable que permite identificar la existencia de ondas de montaña. En las figuras 7.a y 7.b se aprecian claramente bandas alternas de corrientes verticales ascendentes y descendentes, alcanzando hasta $6000 \mathrm{msnm}$, y propagándose unos $40 \mathrm{~km}$ a sotavento de la sierra de Guadarrama y, por tanto, llegando prácticamente hasta el aeropuerto LEMD. La intensidad de las corrientes verticales supera $\operatorname{los} \pm 2 \mathrm{~m} / \mathrm{s}$, pudiendo causar turbulencia moderada durante las maniobras de aproximación y salida (Bolgiani et al., 2018). A primera hora del 12 de diciembre de 2017 (figura 7.c) se aprecian ondas de montaña a sotavento de la sierra de Guadarrama, aunque menos organizadas y de menor intensidad y propagación que las del día 2 de diciembre de 2017, probablemente debido a la menor intensidad de viento y condiciones de estabilidad estática menos favorables. Por la tarde, no se forman ondas de montaña, posiblemente debido a la baja velocidad del viento a $2500 \mathrm{msnm}$.

Posteriormente se ha analizado la humedad relativa siguiendo el mismo corte vertical en el dominio 3 del modelo WRF. Durante el día 2 de diciembre de 2017 (figuras 8.a y 8.b) se aprecia una capa húmeda por debajo de los 3000 msnm a barlovento de la sierra de Guadarrama, mientras que a sotavento se forman bandas alternas con humedades relativas elevadas (incluso superiores a $95 \%$ ) entre 1500 y $3500 \mathrm{msnm}$ coincidiendo con las corrientes ascendentes asociadas a las ondas de montaña (por enfriamiento adiabático), mientras que en las regiones donde predominan las corrientes descendentes la humedad es muy baja (por 

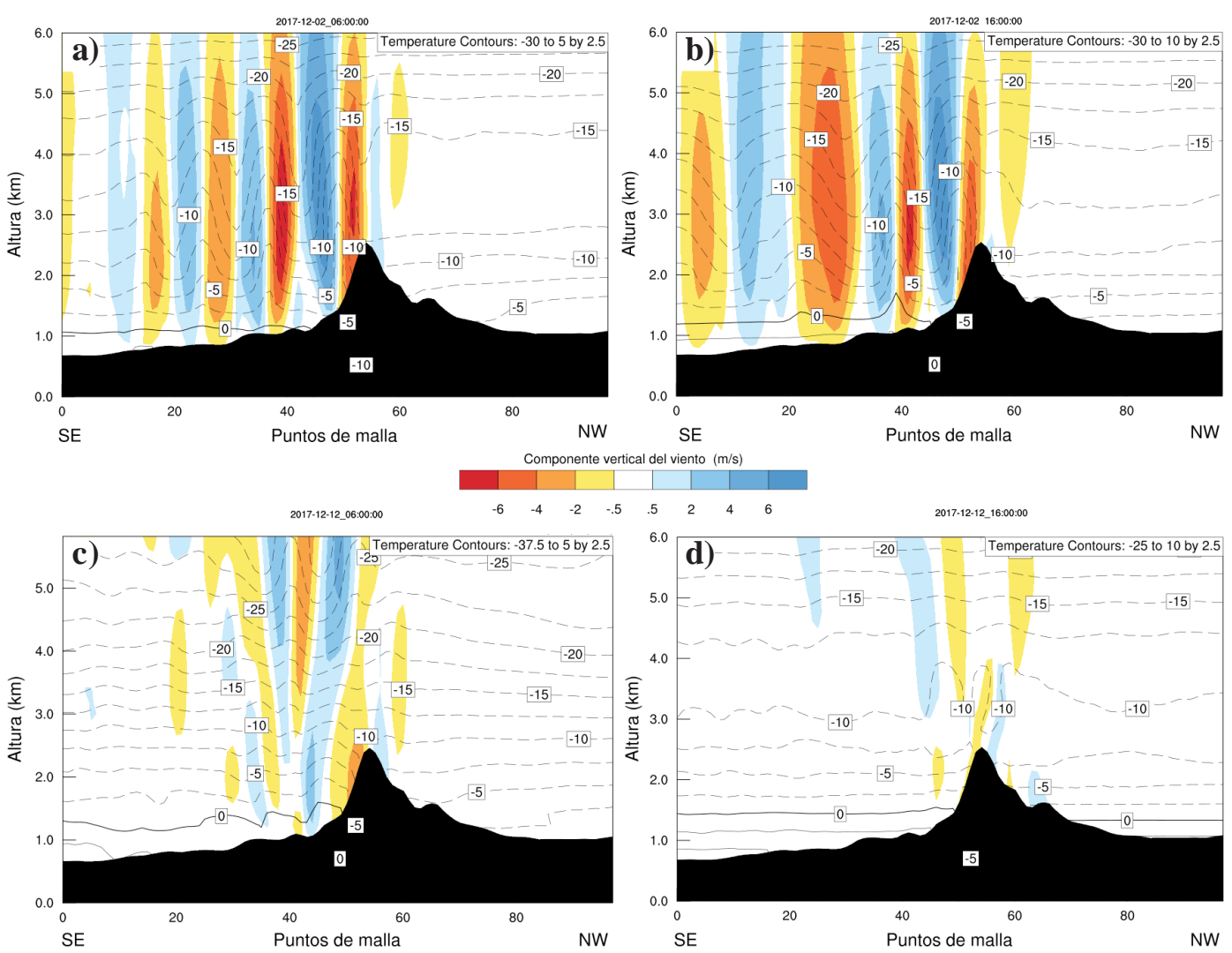

Figura 7. Corte vertical de la componente vertical del viento simulada por el modelo WRF en el dominio 3 a las 06:00 y 16:00 UTC del día 2 de diciembre de 2017 (a y b, respectivamente), y a las 06:00 y 16:00 UTC del día 12 de diciembre de 2017 (c y d).
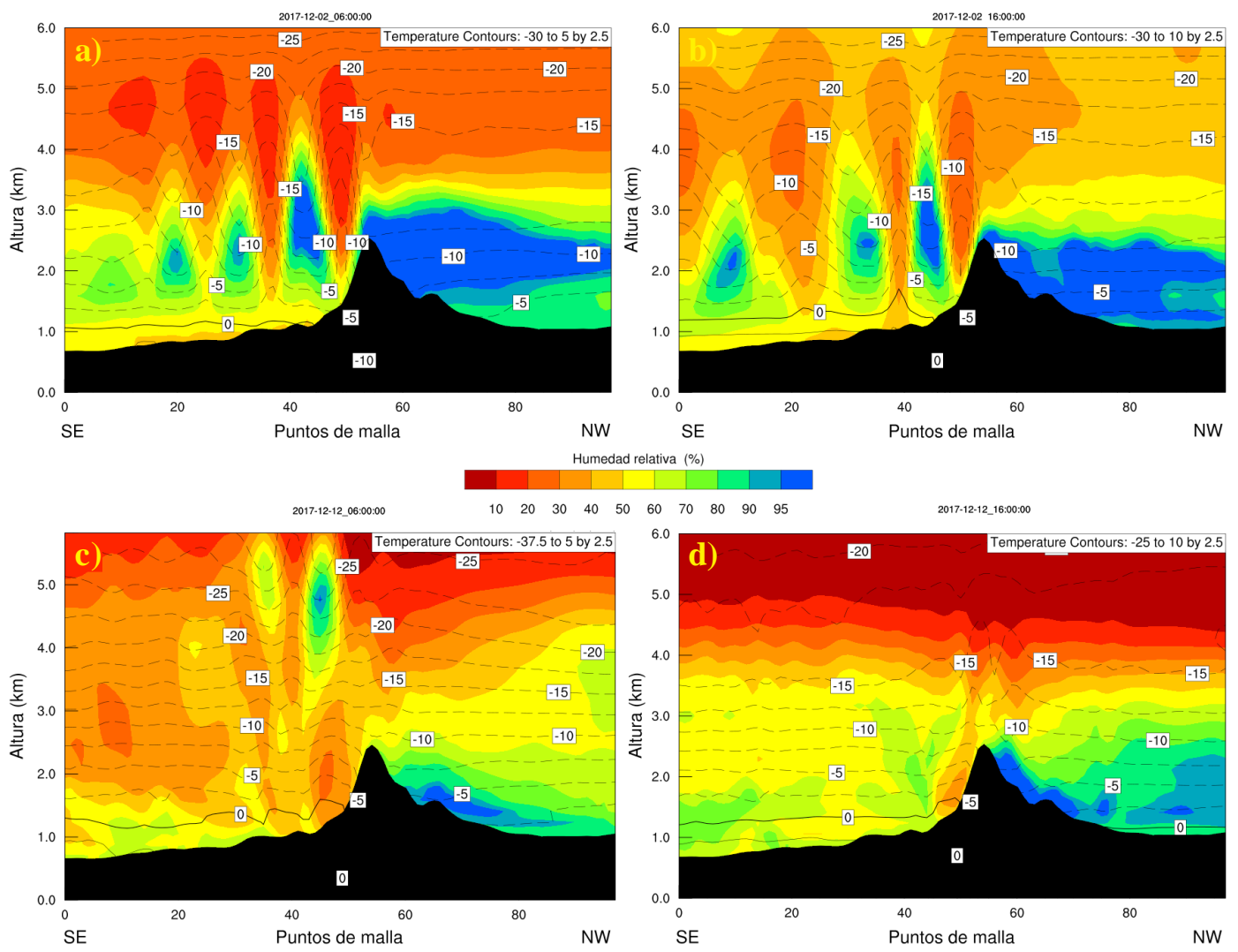

Figura 8. Corte vertical de la humedad relativa a las 06:00 y 16:00 UTC del día 2 de diciembre de 2017 (a y b), y a las 06:00 y 16:00 UTC del día 12 de diciembre de 2017 (c y d). 

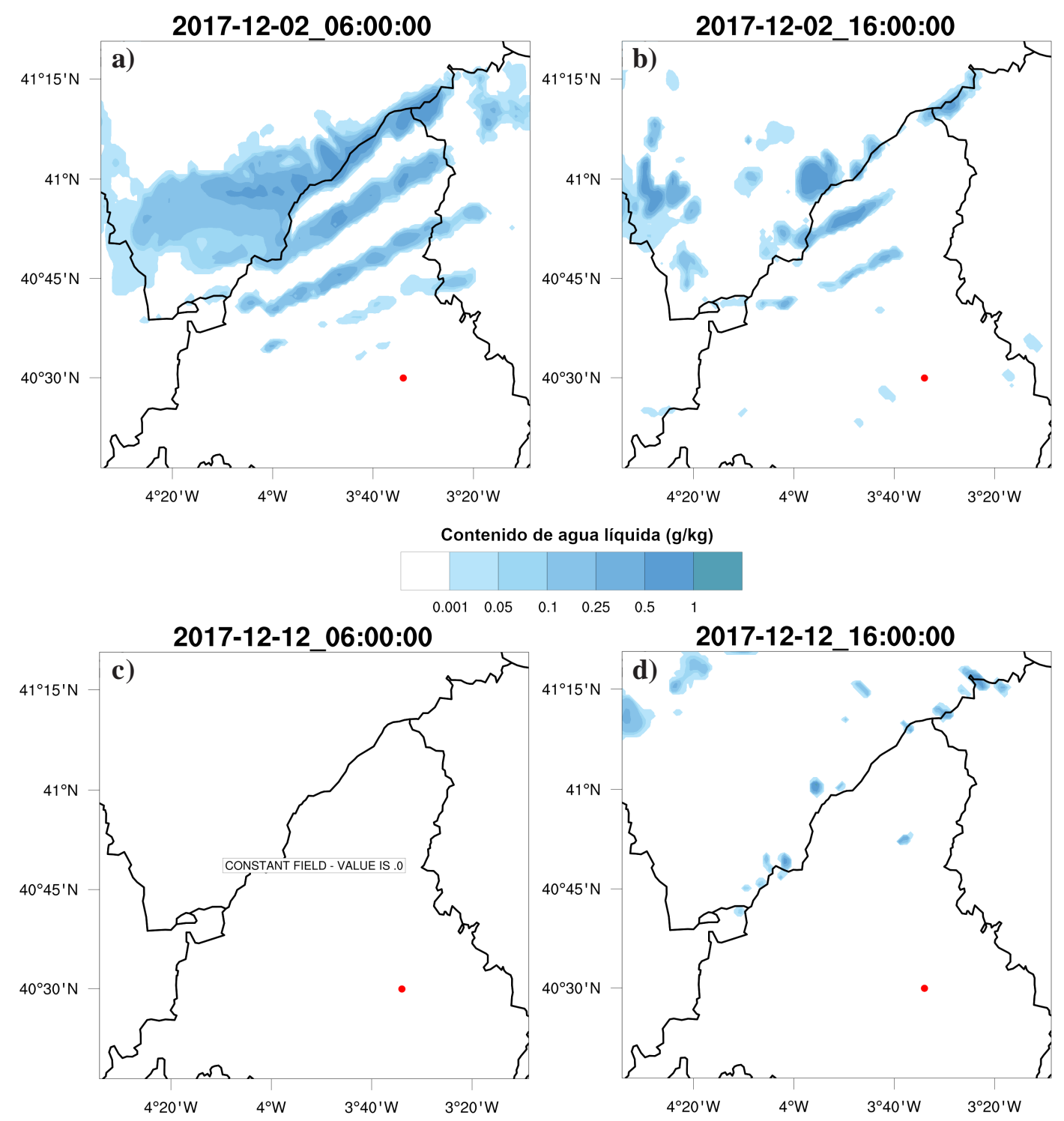

Figura 9. Contenido de agua líquida a $2500 \mathrm{msnm}$ a las 06:00 y 16:00 UTC

del día 2 de diciembre de 2017 (a y b), y a las 06:00 y 16:00 UTC del día 12 de diciembre de 2017 (c y d).

El punto rojo representa la localización del aeropuerto LEMD.

calentamiento adiabático). El día 12 de diciembre de 2017 la capa húmeda a barlovento se limita a los primeros $2000 \mathrm{msnm}$, mientras que a sotavento a primera hora (figura 8.c) solo aparecen 2 bandas con humedad elevada en torno a $4000 \mathrm{msnm}$ (donde la estabilidad estática era mayor), desapareciendo por la tarde (figura 8.d).

Finalmente, se ha analizado el contenido de agua líquida a $2500 \mathrm{msnm}$. A lo largo del día 2 de diciembre de 2017 (figuras 9.a y 9.b) se aprecian bandas con contenido de agua líquida superior a $0,25 \mathrm{~g} / \mathrm{kg}$ a sotavento de la sierra de Guadarrama y paralelas a la misma, que podrían dar lugar a engelamiento moderado al registrarse a esa altura unos $-10^{\circ} \mathrm{C}$. Durante el día 12 de diciembre de 2017 no se aprecia contenido de agua líquida asociado a ondas de montaña, lo que es coherente con la observación realizada por satélite. El modelo WRF estima que el contenido de agua en las ondas de montaña simuladas durante estos episodios se encuentra totalmente en fase líquida, por lo que existe riesgo de engelamiento. Esto puede ser debido a que la eficiencia de los procesos de nucleación para formar cristales de hielo es baja a temperaturas superiores a $-15{ }^{\circ} \mathrm{C}$ (HufFman y Norman Jr., 1988), unido al bajo tiempo de residencia de los hidrometeoros en las ondas de montaña (Korolev y ISAAC, 2000). 


\section{CONCLUSIONES}

La formación de ondas de montaña en las cercanías de aeropuertos es un tema de gran interés debido a la turbulencia y riesgo de engelamiento que pueden causar sobre las aeronaves, especialmente en las maniobras de aproximación y salida. Sin embargo, el pronóstico de ondas de montaña sigue siendo un reto debido a la complejidad de su correcta simulación por parte de los modelos meteorológicos. Por ello, una adecuada caracterización de las condiciones meteorológicas bajo las cuales se forman las ondas de montaña es de vital importancia, con el fin de utilizar esta información para mejorar el pronóstico de futuros episodios. Las principales conclusiones extraídas de este estudio son:

- El canal HRV del satélite METEOSAT permite detectar con precisión la formación de nubosidad asociada a ondas de montaña durante el periodo diurno. Por otra parte, el canal de 7,3 $\mu \mathrm{m}$ es capaz de detectar ondas de montaña de noche e incluso cuando no se forma nubosidad. Además, la composición de colores day natural colors permite discernir la fase en la que se encuentra el techo nuboso, pudiendo alertar de riesgo de engelamiento. Por tanto, estas tres herramientas de teledetección pueden ser de gran utilidad para alertar en tiempo real del riesgo de turbulencia y posible engelamiento.

- Para que se formen ondas de montaña a sotavento de la sierra de Guadarrama se requieren vientos a 2500 msnm de componente NW (aunque también se pueden formar con viento de componente $\mathrm{W}$ y $\mathrm{N}$ ) con velocidades superiores a $20 \mathrm{kt}$.

- Igualmente, es necesario que existan condiciones de ligera estabilidad estática (con valores entre 0,05 y $0,1 \mathrm{~K} / \mathrm{Pa}$ ) para que las ondas puedan propagarse a sotavento.

- Cuando existen condiciones de viento y estabilidad estática favorables, se pueden formar corrientes verticales con una intensidad de $\pm 2 \mathrm{~m} / \mathrm{s}$, que pueden causar turbulencia a las aeronaves que atraviesen la región afectada por ondas de montaña.

- En el caso de que la humedad relativa se encuentre próxima a la saturación existirá riesgo de que se forme nubosidad asociada a las ondas de montaña, con riesgo de engelamiento en caso de encontrarnos por encima de la isocero.

En definitiva, en este trabajo se propone el uso de distintos canales del satélite MSG y simulaciones mediante modelos mesoescalares para mejorar el pronóstico de las ondas de montaña, con el fin de incrementar la seguridad aérea en las proximidades de los aeropuertos. La caracterización de las condiciones meteorológicas relacionadas con la formación de ondas de montaña realizada en este estudio puede servir para identificar futuros episodios, y así alertar a tiempo y estar preparados ante los riesgos de turbulencia y posible engelamiento que puedan afectar a las aeronaves que atraviesen esa zona, pudiendo incluso recomendar que se eviten las regiones con mayor riesgo.

\section{AGRADECIMIENTOS}

Este trabajo ha sido posible gracias a los proyectos TECOAGUA, METEORISK (RTC-2014-1872-5), UE ERA-NET Plus NEWA Project (PCIN-2014-013-C07-04 y PCIN-2016-080) y SAFE-FLIGHT (CGL2016-78702-C2).

\section{REFERENCIAS}

AlLEN, S. J. y VINCENT, R. A., 1995. Gravity wave activity in the lower atmosphere: seasonal and latitudinal variations. J. Geophys. Res., 100, 1327-1350. 
Bernstein, B. C., McDonough, F., Politovich, M. K. et al., 2005. Current icing potential: algorithm description and comparison with aircraft observations. J. Appl. Meteorol., 44, 969-986.

Bolgiani, P., Fernández González, S., Martin, M. L., Valero, F., Merino, A., García Ortega, E. y SÁNCHEZ, J. L., 2018. Analysis and numerical simulation of an aircraft icing episode near Adolfo Suárez Madrid-Barajas International Airport. Atmos. Res., 200, pp. 60-69.

Broutman, D., Rottman, J. W., Eckermann, S. D. y Hulburt, E. O., 2001. A hybrid method for wave propagation from a localized source, with application to mountain waves. Quarterly Journal of the Royal Meteorological Society, 127 (571), 129-146.

DudHIA, J., 1989. Numerical study of convection observed during the winter monsoon experiment using a mesoscale two-dimensional model. J. Atmos. Sci., 46 (20), 3077-3107.

Fernández González, S., Sánchez, J. L., Gascón, E., López, L., García Ortega, E. y Merino, A., 2014. Weather features associated with aircraft icing conditions: a case study. Sci. World J., 1-19.

Huffman, G. J. y Norman JR., G. A., 1988. The supercooled warm rain process and the specification of freezing precipitation. Monthly Weather Review, 116(11), 2172-2182.

KaIN, J., 2004. The Kain-Fritsch convective parameterization: an update. J. Appl. Meteorol., 43 (1), 170-181.

Korolev, A. V. y IsAac, G. A., 2000. Drop growth due to high supersaturation caused by isobaric mixing. Journal of the Atmospheric Sciences, 57 (10), 1675-1685.

LENSKY, I. M. y RosenFeld, D., 2008. Clouds-aerosols-precipitation satellite analysis tool (CAPSAT). Atmos. Chem. Phys., 8 (22), 6739-6753.

Mlawer, E., Taubman, S., Brown, P., Iacono, M. y Clough, S., 1997. Radiative transfer for inhomogeneous atmospheres: RRTM, a validated correlated-k model for the longwave. J. Geophys. Res., 102 (14), 16663-16682.

Moreno, M. C. y GiL, M. A., 2003. Análisis de la siniestralidad aérea por causa meteorológica (1970-1999). Investigaciones geográficas, 30, 7-25.

NAKANISHI, M. y NiINO, H., 2006. An improved Mellor-Yamada level-3 model: its numerical stability and application to a regional prediction of advection fog. Bound.-Layer Meteorol., 119, 397-407.

Petersen, G. N., Kristuánsson, J. E. y Ólafsson, H., 2005. The effect of upstream wind direction on atmospheric flow in the vicinity of a large mountain. Quarterly Journal of the Royal Meteorological Society, 131 (607), 1113-1128.

Setvák, M., Rabin, R. M., Doswell III, C. A. y Levizzani, V., 2003. Satellite observations of convective storm tops in the 1.6, 3.7 and $3.9 \mu \mathrm{m}$ spectral bands. Atmos. Res., 67-68, 607-627.

SkAmarock, W. y KLEMP, J., 2008. A time-split nonhydrostatic atmospheric model for weather research and forecasting applications. J. Comput. Phys., 227 (7), 3465-3485.

Smirnova, T. G., Brown, J. M. y Kim, D., 2000. Parameterization of cold-season processes in the MAPS land-surface scheme. J. Geophys. Res., 105, 4077-4086.

Thompson, G., Field, P., Rasmussen, R. y Hall, W., 2008. Explicit forecasts of winter precipitation using an improved bulk microphysics scheme. Part II: implementation of a new snow parameterization. Mon. Weather Rev., $136(12)$, 5095-5115. 